\title{
Risks and Benefits of Artificial Intelligence in Teledermatology
}

\author{
Josep Malvehy, $\mathrm{PhD}$ \\ Dermatology Department, Hospital Clinic of Barcelona, Institut de Investigació August Pi i Sunyer, Universitat de Barcelona, Barcelona, Spain
}

\section{Corresponding Author:}

Josep Malvehy, $\mathrm{PhD}$

Dermatology Department

Hospital Clinic of Barcelona, Institut de Investigació August Pi i Sunyer

Universitat de Barcelona

C de Villarroel, 170

Barcelona, 08036

Spain

Phone: 34932275400

Email: jmalvehy@clinic.cat

\section{Abstract}

Background: Recently, deep convolutional neural networks (DCNNs) became of interest as decision support systems for dermoscopic and clinical analysis of skin diseases. Application of artificial intelligence in teledermatology (TD) has been recently reported in several studies as a tool for augmented intelligence.

Objective: In this session, a critical discussion of the opportunities, limitations, and risks of AI in TD will be presented with special attention to recent published studies.

Methods: We reviewed the literature in PubMed and EMBASE databases in the period of January 2018 to November 2021 with the search terms of dermatology, skin cancer, deep learning, and AI (review of the Regulation of Medical Devices, EU 2017/745).

Results: A clear definition of the clinical use of AI in TD has to be considered: primary TD from patients to nurses, primary care physicians or general dermatologists; secondary TD from primary care physicians or nurses to dermatologists; or tertiary TD from dermatologists to hospital dermatologists. In some health models of TD for nurses or primary care physicians, AI assistance can lower the rates of recommending a biopsy or specialist referral, increase self-reported diagnostic confidence, and help to achieve higher diagnostic agreement rates (with dermatologists) in nonreferred cases. The main limitations of the use of $\mathrm{AI}$ in TD are the lack of large longitudinal studies, the lack of interpretability of the CNN, biases in the databases and unrepresented dermatological conditions for training, limited representation of different ethnicities, standardization of clinical information and of the images, liability, and privacy issues. How to implement the concept of augmented intelligence in clinical practice with referral TD consultations including structured clinical information and good-quality images will need further research and education among end users. Even if the interface is used for either store-and-forward or live TD, interactive TD is, in principle, straightforward for AI systems, and different TD modalities have particular technological requirements that can reduce their efficacy. Finally, AI systems in TD are under the umbrella of medical device regulatory frames, and specific certification is compulsory. This regulation has the benefit of assuring the quality of the new AI systems and diminishing their risks, but it can simultaneously delay the incorporation of AI tools in clinical practice.

Conclusions: AI has the potential to improve the results of the technology in different aspects in multiple modalities of TD. However, the evidence is weak, and several barriers and limitations have to be resolved for their integration in clinical practice.

Conflicts of Interest: None declared.

(iproc 2022;8(1):e36891) doi: 10.2196/36891

\section{KEYWORDS}

artificial intelligence; deep learning; convolutional neural networks; teledermatology; risks; benefits 
Edited by T Derrick; this is a non-peer-reviewed article. Submitted 28.01.22; accepted 28.01.22; published 18.02.22.

Please cite as:

Malvehy $J$

Risks and Benefits of Artificial Intelligence in Teledermatology

iproc 2022;8(1):e36891

URL: https://www.iproc.org/2022/1/e36891

doi: $\underline{10.2196 / 36891}$

PMID:

CJosep Malvehy. Originally published in Iproceedings (https://www.iproc.org), 18.02.2022. This is an open-access article distributed under the terms of the Creative Commons Attribution License (https://creativecommons.org/licenses/by/4.0/), which permits unrestricted use, distribution, and reproduction in any medium, provided the original work, first published in Iproceedings, is properly cited. The complete bibliographic information, a link to the original publication on https://www.iproc.org/, as well as this copyright and license information must be included. 